\title{
THE INTERPLANETARY NETWORK RESPONSE TO LIGO GW150914
}

\author{
K. Hurley ${ }^{1}$, D. S. Svinkin ${ }^{2}$, R. L. Aptekar ${ }^{2}$, S. V. Golennetskit ${ }^{2}$, D. D. Frederiks ${ }^{2}$, W. Boynton ${ }^{3}$, I. G. Mitrofanov ${ }^{4}$, \\ D. V. Golovin ${ }^{4}$, A. S. KozYrev ${ }^{4}$, M. L. LitvaK $^{4}$, A. B. SAnin $^{4}$, A. Rau ${ }^{5}$, A. von Kienlin ${ }^{5}$, X. Zhang ${ }^{5}$, V. Connaughton ${ }^{6}$,

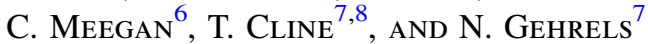 \\ ${ }^{1}$ University of California, Berkeley, Space Sciences Laboratory, 7 Gauss Way, Berkeley, CA 94720-7450, USA; khurley@ssl.berkeley.edu \\ ${ }^{2}$ Ioffe Physical Technical Institute, Politekhnicheskaya 26, St. Petersburg 194021, Russia \\ ${ }^{3}$ University of Arizona, Department of Planetary Sciences, Tucson, AZ 85721, USA \\ ${ }^{4}$ Space Research Institute, 84/32, Profsoyuznaya, Moscow 117997, Russia \\ ${ }^{5}$ Max-Planck-Institut für extraterrestrische Physik, Giessenbachstrasse, Postfach 1312, Garching, D-85748 Germany \\ ${ }^{6}$ University of Alabama in Huntsville, NSSTC, 320 Sparkman Drive, Huntsville, AL 35805, USA \\ NASA Goddard Space Flight Center, Code 661, Greenbelt, MD 20771, USA \\ Received 2016 May 5; revised 2016 July 20; accepted 2016 August 1; published 2016 September 19
}

\begin{abstract}
We have performed a blind search for a gamma-ray transient of arbitrary duration and energy spectrum around the time of the LIGO gravitational-wave event GW150914 with the six-spacecraft interplanetary network (IPN). Four gamma-ray bursts were detected between $30 \mathrm{hr}$ prior to the event and $6.1 \mathrm{hr}$ after it, but none could convincingly be associated with GW150914. No other transients were detected down to limiting 15-150 keV fluences of roughly $5 \times 10^{-8}-5 \times 10^{-7} \mathrm{erg} \mathrm{cm}^{-2}$. We discuss the search strategies and temporal coverage of the IPN on the day of the event and compare the spatial coverage to the region where GW150914 originated. We also report the negative result of a targeted search for the Fermi-GBM event reported in conjunction with GW150914.
\end{abstract}

Key words: gamma-ray burst: general - gravitational waves

\section{INTRODUCTION}

The interplanetary network (IPN) presently comprises six experiments on board six spacecraft with orbits that range from near-Earth to Martian. They are: Konus-Wind, at distances up to around 5 lt-s from Earth (Aptekar et al. 1995); Mars Odyssey HEND, in orbit around Mars at up to 1250 lt-s from Earth (Hurley et al. 2006); the International Gamma-ray Laboratory (INTEGRAL SPI-ACS), in an eccentric Earth orbit at up to 0.5 lt-s from Earth (Rau et al. 2005); and RHESSI (Smith et al. 2002), Swift-BAT (Gehrels et al. 2004), and Fermi-GBM (Meegan et al. 2009), all in low Earth orbit. The detection of a gamma-ray burst or other transient by two or more instruments can be used to constrain its source location by triangulation if the detectors are separated by more than a few light-seconds. Individually, the experiments have varying duty cycles and anisotropic responses, and some sky positions are subject to intermittent planetary occultation for some of them; however, when taken as an ensemble, the IPN is effectively an all-sky, full-time monitor. Thus, it is particularly well-suited to the search for gamma-ray transients in response to particular events, such as the LIGO gravitational-wave transient GW150914 (Abbott et al. 2016a). Indeed, the IPN has been collaborating with the LIGO project since some of its earliest engineering runs in 2001.

A brief description of the IPN search for a gamma-ray transient associated with GW150914 was given in Abbott et al. (2016b). Here, we describe the mission characteristics in more detail and present the results of both a blind and a targeted search.

\section{MISSION CHARACTERISTICS}

The experiments in the IPN share the fact that they detect gamma-rays with good sensitivity over a wide energy range,

\footnotetext{
${ }^{8}$ Emeritus.
}

with very precise timing. However, they vary widely in their effective areas, shapes, volumes, and detector materials. Konus, BAT, and GBM are dedicated GRB detectors that produce both triggered burst data and continuous streams of untriggered data that can be searched for bursts that do not meet the trigger criteria; both are utilized in the IPN, but the BAT data used are primarily for bursts outside its coded field of view, which are generally untriggered. HEND, SPI-ACS, and RHESSI are not dedicated GRB detectors, but they produce continuous streams of high time resolution data that are searched in ground software for transients. (An independent analysis of the SPIACS response is discussed in detail in Savchenko et al. 2016.) The instrument sensitivities differ widely due to their different background environments, energy ranges, and effective areas; in addition, experiments may be more or less sensitive to GRBs with short or long time histories, or with harder or softer energy spectra. BAT, GBM, and RHESSI have duty cycles that are primarily governed by their passage through the South Atlantic Anomaly; the duty cycles of Konus, HEND, and SPI-ACS, on the other hand, are higher and mainly limited by available telemetry downlinks. A final consideration is that each instrument has a different anisotropic response, due to factors such as detector shape, collimation, shielding by surrounding spacecraft materials, and, for BAT, GBM, HEND, and RHESSI, planet-blocking. These differences make it complex to describe the IPN characteristics in detail. That is beyond the scope of this Letter and indeed is not a primary consideration for the present search; instead, Table 1 summarizes the essentials. For each experiment, Table 1 gives the following information.

1. The spacecraft and/or instrument.

2. The orbit; altitude above the planetary surface and the inclination are given for BAT, GBM, RHESSI, and HEND. The inclination determines the region of R.A. and decl. that is intermittently occulted. Because HEND is in 
Table 1

Characteristics of the IPN Missions and Experiments

\begin{tabular}{|c|c|c|c|c|c|c|}
\hline Instrument & Orbit & $\begin{array}{c}\text { Maximum Sky } \\
\text { Coverage, } \%\end{array}$ & $\begin{array}{c}\text { Duty Cycle, } \\
\%\end{array}$ & $\begin{array}{c}\text { Energy Range, } \\
\text { keV }\end{array}$ & $\begin{array}{l}\text { Approx. Sensitivities, } \\
\mathrm{erg} \mathrm{cm}^{-2}\end{array}$ & $\begin{array}{c}\text { Number of } \\
\text { GRBs } / y\end{array}$ \\
\hline Odyssey HEND & $400 \mathrm{~km}, 93.1$ & 72 & 86 & $50-3000$ & $7 \times 10^{-8}, 2 \times 10^{-7}$ & 67 \\
\hline RHESSI & $600 \mathrm{~km}, 38^{\circ}$ & 69 & 59 & $30-150$ & $5 \times 10^{-8}, 1.5 \times 10^{-7}$ & 69 \\
\hline Swift-BAT & $600 \mathrm{~km}, 20.6$ & 69 & 86 & $15-150$ & $10^{-7}, 5.3 \times 10^{-7}$ & 83 \\
\hline Konus-Wind & $1.5 \times 10^{6} \mathrm{~km}$ & 100 & 99.4 & $20-1450$ & $7 \times 10^{-8}, 4.3 \times 10^{-7}$ & 169 \\
\hline INTEGRAL SPI-ACS & $1.5 \times 10^{5} \mathrm{~km}, 52.5$ & 100 & 94 & $\gtrsim 75$ & $2.5 \times 10^{-8}, 1.1 \times 10^{-7}$ & 221 \\
\hline Fermi-GBM & $530 \mathrm{~km}, 25.6$ & 69 & 83 & 10-1000 & $2.3 \times 10^{-8}, 2 \times 10^{-7}$ & 248 \\
\hline
\end{tabular}

Note. The following information is given for the IPN instruments: spacecraft and/or experiment, orbital altitude and inclination, sky coverage taking planet-blocking into account, duty cycle on 2015 September 14, nominal experiment energy range, 15-150 keV sensitivities for short- and long-duration bursts, and number of confirmed bursts detected in 2015 .

a Martian polar orbit, and the Martian north pole is oriented toward declination $52^{\circ} .9$, all declinations are intermittently occulted. For BAT, GBM, and RHESSI, there are bands of declination that are never Earthocculted.

3. The maximum sky coverage, in percent, as determined by the orbital altitude for RHESSI, Swift, Odyssey, and Fermi; the planetary equatorial radius has been used, and the effects of the planetary atmospheres are not taken into account. Earth-blocking is negligible for INTEGRAL at apogee, and for Wind.

4. The duty cycle; this is the percentage of the time that usable data were recovered on 2015 September 14. For Konus and SPI-ACS, which have stable backgrounds, this is simply the percentage of data received. For the other missions, whose backgrounds are variable, this is the percentage of data received where the count rate in Figure 1 is nonzero and less than 500 (RHESSI), 15,000 (Swift), 700 (Odyssey), or 4000 (Fermi). Although a sufficiently strong event can be detected at any background level, these numbers represent reasonable estimates of the maximum usable rates, and the duty cycles, although variable, can be considered typical for other days as well.

5. The nominal energy range of the instrument, in $\mathrm{keV}$.

6. The approximate sensitivities, given in fluence and normalized to the $15-150 \mathrm{keV}$ energy band (which partially or completely overlaps the energy ranges of all the IPN instruments), to allow comparisons between missions. The first number is for the weakest shortduration $(<1 \mathrm{~s})$ burst, and the second number is for the weakest long-duration $(>2 s)$ burst observed by the instrument in question; the durations are also measured in the 15-150 keV range. For Swift-BAT, these numbers are for bursts observed outside the coded field of view. In all cases, these numbers refer to confirmed bursts that were actually detected by the instruments.

7. The number of confirmed bursts of any duration observed by the instruments in 2015. For Swift-BAT, this is the rate of bursts observed outside the coded field of view.

\section{TEMPORAL AND SPATIAL COVERAGE}

Figure 1 shows the count rates of the six IPN instruments on 2015 September 14. The vertical line indicates the time of GW150914 (35,445 s UT). All instruments were taking data under good background conditions at the time. Figure 2 shows the portions of the sky that were Earth-blocked or Marsblocked to RHESSI, Swift, Fermi, and Odyssey at the time of the event, as well as the LIGO 90\% LALinference error box (Abbott et al. 2016b). RHESSI, Konus, INTEGRAL, and Odyssey viewed the entire LIGO region, while Swift and Fermi viewed $99 \%$ and $70 \%$ of it, respectively. (Note that LIGO regions have been derived in several different ways, with several different confidence levels, and that the regions can differ fairly substantially from one another. Also, the Marscrossing time depends on the assumed arrival direction, and has been taken to be the same as the Earth-crossing time in Figure 2.)

\section{RESULTS}

Table 2 summarizes a fairly typical set of search strategies for IPN events. Usually one or more detectors trigger on an event, initiating a search through the data of the other spacecraft on a variety of timescales. If the localization of a burst is known even coarsely (e.g., from Fermi-GBM), planetblocked regions are calculated for RHESSI, Swift, and Odyssey to determine whether the burst could have been detected by them. In this case, for GW150914, the blind search looked for a burst of any duration or spectrum in $\approx 2$ days centered around the time of GW150914. All the searches in Table 2 took place. This search was motivated by three factors. First, there are few predictions for the type of electromagnetic event, if any, that is expected to accompany an event such as GW150914. Second, although Fermi has reported a burst associated with GW150914 (Connaughton et al. 2016) and explanations for it have been proposed (Loeb 2016; Woosley 2016), roughly 30\% of the LIGO error box was Earth-blocked to it, leaving open the possibility that other electromagnetic transients could also be present. And third, although the results of an INTEGRAL search have been reported in Savchenko et al. (2016), the lack of coverage below $75 \mathrm{keV}$ for the SPI-ACS instrument means that a softer spectrum transient could have been missed.

In addition to the blind search, a targeted search specifically for the Fermi-GBM event, a $1 \mathrm{~s}$ long untriggered burst with a hard spectrum that was found in time-tagged event data, was also carried out.

The results of the blind search may be summarized as follows. The IPN bursts that were detected before and after GW150914 occurred at 2015 September 13 13,917 s UT (30 hr before), 2015 September 14 24,549 s UT (3 hr before), 2015 September 14 49,358 s (3.9 hr after), and at 2015 September 14 $57,365 \mathrm{~s}$ UT (6.1 hr after). All were long-duration GRBs. The first burst was detected by Odyssey, Konus, RHESSI, 


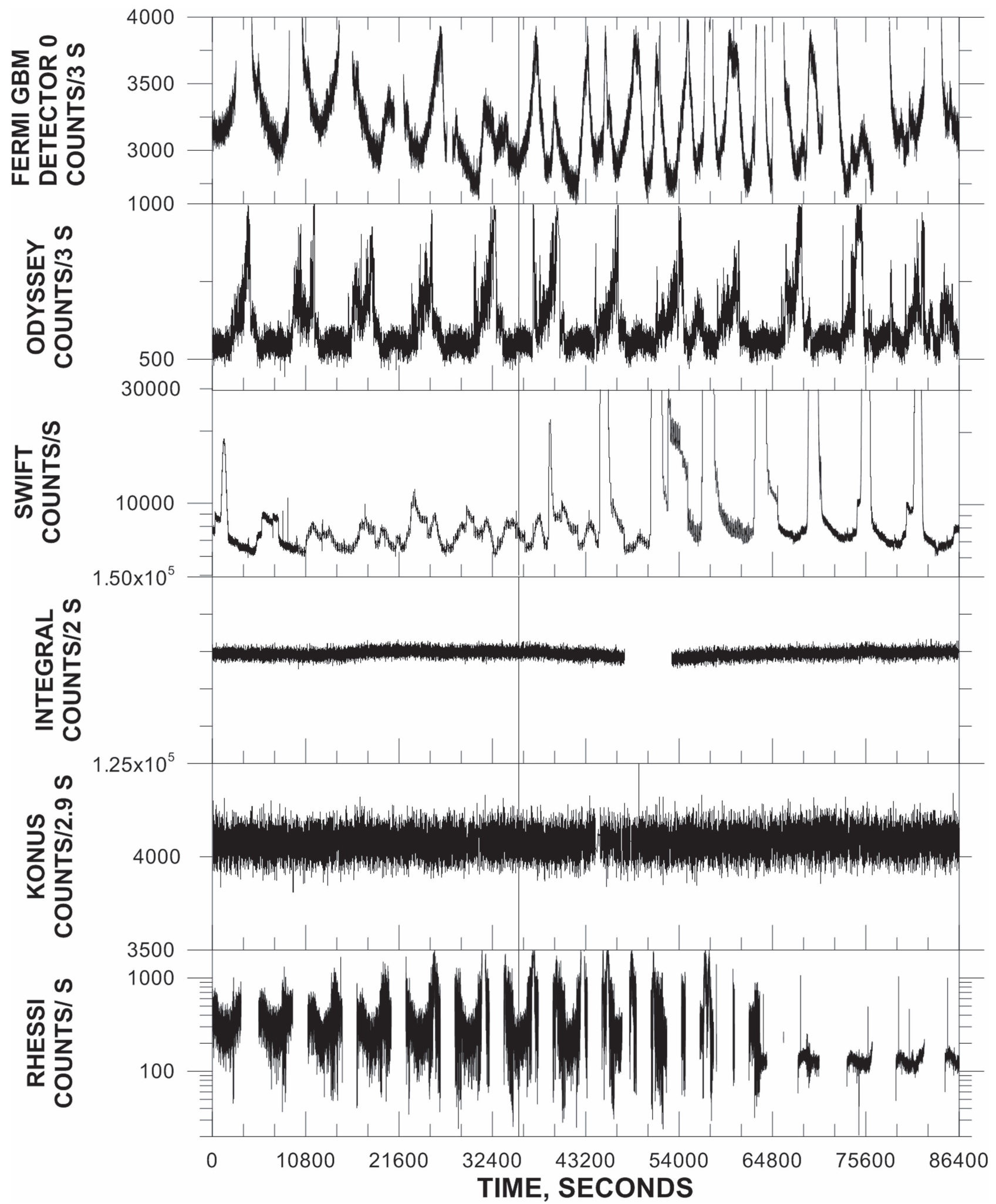

Figure 1. Count rates of the six IPN instruments for the day of 2015 September 14. For clarity, coarse time resolutions have been plotted for a single detector in the cases of Konus and Fermi. All the IPN instruments have much finer time resolution. The vertical line indicates the time of GW150914. All instruments were taking data under good background conditions at the time of the event.

INTEGRAL, Swift (outside the coded field of view), and Fermi, and had a triangulated arrival direction around $\alpha=234^{\circ} .2, \delta=-53.7$, which is clearly inconsistent with the LIGO region. The second, third, and fourth events were detected only in Konus untriggered (waiting mode) data with approximately $10 \sigma$ significance. The second event has an arrival direction in the north ecliptic hemisphere at a latitude above $30^{\circ}$, which is also inconsistent with the LIGO event. The 


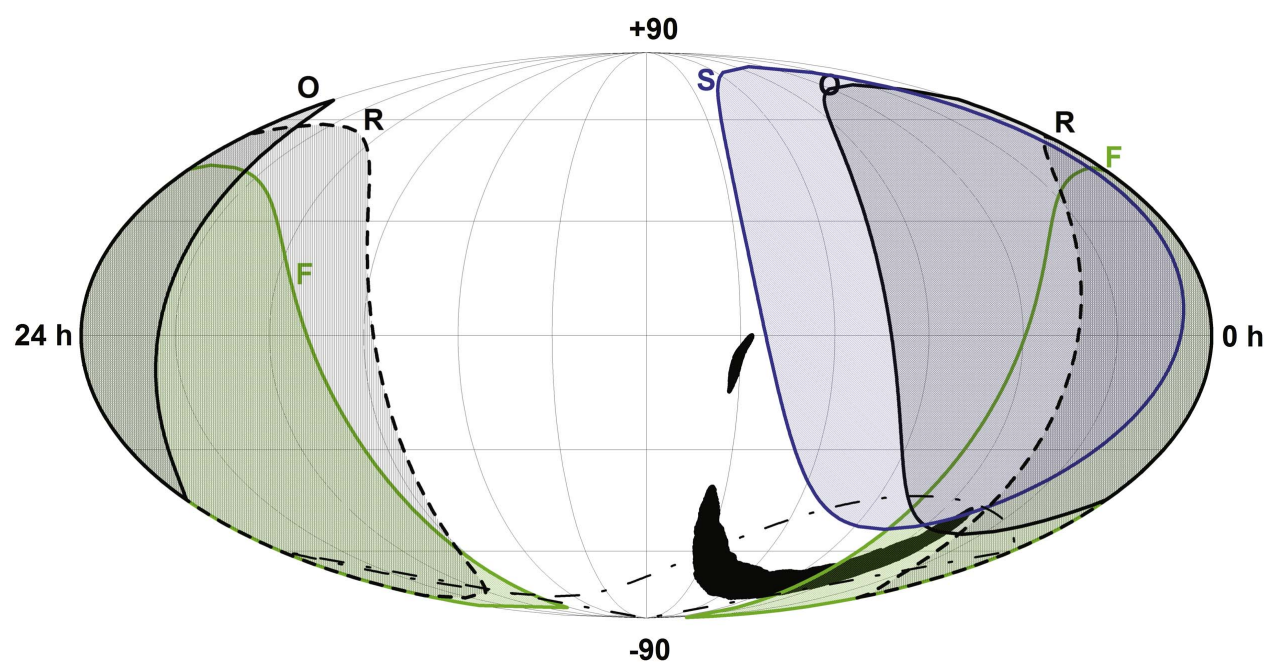

Figure 2. Mollweide projection showing the regions of the sky that were Earth- or Mars-blocked to RHESSI (R, dashed line, black fill), Swift (S, blue fill), Fermi ( $F$, green fill), and Odyssey (O, black fill). INTEGRAL and Konus viewed virtually the entire sky. The LIGO 90\% LALInference error box for GW150914 is shown in black. About $30 \%$ of this region is Earth-blocked to Fermi, and about $1 \%$ to Swift. The dotted-dashed line indicates the Fermi-GBM error box, about $42 \%$ of which is below the Fermi horizon.

Table 2

IPN Search Strategies

\begin{tabular}{|c|c|c|c|c|c|c|}
\hline \multirow[b]{2}{*}{ Data Searched } & \multicolumn{6}{|c|}{ Initiating Instrument } \\
\hline & Fermi & INTEGRAL & Konus & Odyssey & RHESSI & Swift \\
\hline Fermi $^{\mathrm{a}}$ & $\cdots$ & Triggers & Triggers & Triggers & Triggers & Triggers \\
\hline INTEGRAL SPI-ACS ${ }^{\mathrm{b}}$ & $0.05,0.5,5 \mathrm{~s}$ data & $\ldots$ & $0.05,0.5,5 \mathrm{~s}$ data & $0.05,0.5,5 \mathrm{~s}$ data & $0.05,0.5,5 \mathrm{~s}$ data & $0.05,0.5,5 \mathrm{~s}$ data \\
\hline Konus $^{\mathrm{c}}$ & Triggers, $2.9 \mathrm{~s}$ & Triggers, $2.9 \mathrm{~s}$ & $\ldots$ & Triggers, $2.9 \mathrm{~s}$ & Triggers, $2.9 \mathrm{~s}$ & Triggers, $2.9 \mathrm{~s}$ \\
\hline Odyssey $^{\mathrm{d}}$ & $.25 \mathrm{~s}$ data & $.25 \mathrm{~s}$ data & $.25 \mathrm{~s}$ data & $\ldots$ & $.25 \mathrm{~s}$ data & $.25 \mathrm{~s}$ data \\
\hline RHESSI & $0.05,0.5,5 \mathrm{~s}$ & $0.05,0.5,5 \mathrm{~s}$ & $0.05,0.5,5 \mathrm{~s}$ & $0.05,0.5,5 \mathrm{~s}$ & $\ldots$ & $0.05,0.5,5 \mathrm{~s}$ \\
\hline Swift-BAT ${ }^{\mathrm{f}}$ & $0.064 \mathrm{~s}$ & $0.064 \mathrm{~s}$ & $0.064 \mathrm{~s}$ & $0.064 \mathrm{~s}$ & $0.064 \mathrm{~s}$ & $\ldots$ \\
\hline
\end{tabular}

Notes. For each instrument that reports a possible burst, the table describes the types of data that are searched in the other IPN instruments for a confirmation.

${ }^{a}$ GBM data are both triggered and time-tagged event; typically, only triggers are examined.

${ }^{\mathrm{b}}$ INTEGRAL SPI-ACS data consist of a continuous stream of $0.050 \mathrm{~s}$ count rates in a single energy range; they are regrouped to produce 0.5 and $5 \mathrm{~s}$ rates.

${ }^{\mathrm{c}}$ Konus data are both triggered, and waiting mode; waiting mode data have $2.944 \mathrm{~s}$ resolution. Both are searched.

d Odyssey HEND data consist of a continuous stream of $0.25 \mathrm{~s}$ count rates in a single energy range. If the burst arrival direction is known, a specific Mars-crossing window is calculated and examined. If not, the Mars-crossing window is plus or minus the light-travel time between Earth and Mars.

${ }^{\mathrm{e}}$ RHESSI data consist of time-tagged events. They are typically searched in the $30-150 \mathrm{keV}$ energy range on three timescales spanning two orders of magnitude, tailored to the burst duration.

${ }_{\mathrm{f}}^{\mathrm{f}}$ Swift-BAT data outside the coded field of view consist of time-tagged events. They are typically searched in the $15-350 \mathrm{keV}$ energy range on a $0.064 \mathrm{~s}$ timescale.

third event can be localized only to the south ecliptic hemisphere at latitude $>24^{\circ}$; the probability of including any point on the sky by chance is about 0.3 . The fourth event can be localized to the north ecliptic hemisphere, which is inconsistent with the LIGO event.

Short-duration and long-duration bursts occurring between the times of the first and fourth bursts could have been detected down to the sensitivity limits given in Table 1. These limits are general and do not apply to bursts with any particular spectral type. More specifically, if we assume a typical Konus short GRB spectrum (an exponentially cut off power law, or CPL, $\propto E^{\alpha} \exp \left(-E(2+\alpha) / E_{p}\right)$ with $\alpha=-0.5$ and $\mathrm{E}_{p}=500 \mathrm{keV}$; Svinkin et al. 2016) we derive $90 \%$ confidence upper limits of $\approx 2 \times 10^{-7} \mathrm{erg} \mathrm{cm}^{-2}(10 \mathrm{keV}-1 \mathrm{MeV}$; the GBM band $)$ and $4 \times 10^{-8} \mathrm{erg} \mathrm{cm}^{-2}(15-150 \mathrm{keV}$; the band used in Table 1).

For the targeted search, no events were detected, and the following limits apply. The weakest detected IPN bursts with durations around $1 \mathrm{~s}$ had approximate $15-150 \mathrm{keV}$ fluences and durations of $8.8 \times 10^{-7}$ (Odyssey, $\left.0.8 \mathrm{~s}\right), 4.2 \times 10^{-7}$ (Konus,
$0.84 \mathrm{~s}), 1.4 \times 10^{-7}$ (Fermi triggered, $\left.1 \mathrm{~s}\right), 1.8 \times 10^{-7}$ (Swift, outside the coded field of view, $0.8 \mathrm{~s}$ ), $1.4 \times 10^{-7}$ (INTEGRAL, $1 \mathrm{~s})$, and $1.2 \times 10^{-6}$ (RHESSI, $\left.1.4 \mathrm{~s}\right)$, all in erg cm${ }^{-2}$. These limits are also general and do not refer to any particular energy spectrum. A more specific search can be carried out using Konus waiting mode data. In this mode, a continuous stream of untriggered count rate data with $2.944 \mathrm{~s}$ resolution is transmitted to the ground for each of the two detectors in three energy bands and can be searched using various algorithms. We obtain a $90 \%$ confidence upper limit to the $10 \mathrm{keV}-$ $10 \mathrm{MeV}$ fluence for a burst with duration $\lesssim 2.944 \mathrm{~s}$ and with the very hard CPL spectrum reported by the GBM $(\alpha=-0.16$ and $E_{p}=3.3 \mathrm{MeV}$ ) of $\approx 2 \times 10^{-6} \mathrm{erg} \mathrm{cm}^{-2}$. This corresponds to $\approx 3 \times 10^{-7} \mathrm{erg} \mathrm{cm}^{-2}$ in the $10 \mathrm{keV}-1 \mathrm{MeV}$ GBM band and $1.5 \times 10^{-8} \mathrm{erg} \mathrm{cm}^{-2}$ in the $15-150 \mathrm{keV}$ band. If instead we use the GBM power-law fit with an index of -1.4 , we obtain $1 \times 10^{-6}(10 \mathrm{keV}-10 \mathrm{MeV}), 2.3 \times 10^{-7}(10 \mathrm{keV}-1 \mathrm{MeV})$, and $6 \times 10^{-8} \mathrm{erg} \mathrm{cm}^{-2}(15-150 \mathrm{keV})$. Alternatively, using the same typical short GRB spectrum given above (CPL, $\propto E^{\alpha} \exp$ 
$\left(-\mathrm{E}(2+\alpha) / E_{p}\right)$ with $\alpha=-0.5$ and $\left.\mathrm{E}_{p}=500 \mathrm{keV}\right)$, we derive the same $90 \%$ confidence upper limits as in the preceding paragraph $\left(\approx 2 \times 10^{-7} \mathrm{erg} \mathrm{cm}^{-2}, 10 \mathrm{keV}-1 \mathrm{MeV}\right.$, and $\left.4 \times 10^{-8} \mathrm{erg} \mathrm{cm}^{-2}, 15-150 \mathrm{keV}\right)$.

Finally, since bursts of this duration can also be detected on the basis of peak flux, rather than fluence, we have investigated whether a $1 \mathrm{~s}$ burst with a peak flux of roughly 0.8 photons $\mathrm{cm}^{-2} \mathrm{~s}^{-1}$ (the estimated GBM $15-150 \mathrm{keV} 1 \mathrm{~s}$ peak flux) could have been detected by the other IPN experiments. No $\approx 1 \mathrm{~s}$ long events with a peak flux this small have been detected to date.

\section{CONCLUSIONS}

Given that the IPN instruments had good spatial and temporal coverage of GW150914, it is plausible that a burst down to the sensitivity limits in Table 1 within the LIGO region could have been detected by them, and indeed, the Fermi GBM did detect such an event in time-tagged event data, as described elsewhere (Connaughton et al. 2016). We have not attempted to repeat that analysis. The fluence of that burst in the $15-150 \mathrm{keV}$ energy range was about $6 \times 10^{-8} \mathrm{erg} \mathrm{cm}^{-2}$. Bursts with fluences less than this and with shorter durations than the GBM event have been detected previously with RHESSI and INTEGRAL. Also, Konus's estimated sensitivity is comparable to the GBM fluence. However, none of the IPN instruments have to date actually detected bursts with the GBM duration of $\approx 1 \mathrm{~s}$ and with lower fluence or peak flux. It should be noted, however, that the detection of bursts close to the threshold depends on other factors such as the precise arrival direction (which may be blocked by intervening spacecraft materials), the exact background conditions (short bursts, in particular, can be masked by short-duration background fluctuations when they are close to the threshold), the shape of the time history, and the energy spectrum. In the blind search, these are mostly unknown or, in the case of the arrival direction, uncertain. In the targeted search it is possible to narrow the parameter space by specifying the duration and spectrum, and reducing the localization uncertainty, but in both cases the lack of detection by other IPN instruments is probably at least as likely as a detection.

Using the conventional data processing methods and data products of the IPN, we did not detect the Fermi-GBM event (Connaughton et al. 2016), nor indeed any other event that could reasonably be associated with GW150914. We believe that the lack of detection of the Fermi event is likely to be due to its weakness, and therefore we cannot comment on its origin or relation to the LIGO event. Nevertheless, the IPN's all-sky, full-time monitoring capabilities make it well-suited to future searches for electromagnetic counterparts to gravitational-wave events.

This research has made use of data, software, and/or web tools obtained from the High Energy Astrophysics Science Archive Research Center (HEASARC), a service of the Astrophysics Science Division at NASA/GSFC and of the Smithsonian Astrophysical Observatory's High Energy Astrophysics Division. We thank Amy Lien for help with the Swift data. K.H. is grateful for support under NASA grant NNX15AU74G. R.L.A. and S.V.G. gratefully acknowledge support from RFBR grant 15-02-00532.

\section{REFERENCES}

Aptekar, R., Frederiks, D., Golenetskii, S., et al. 1995, SSRv, 71, 265 Abbott, B., Abbott, R., Abbott, T., et al. 2016a, PhRvL, 116, 061102 Abbott, B., Abbott, R., Abbott, T., et al. 2016b, PhRvD, 93, 122003 Connaughton, V., Burns, E., Goldstein, A., et al. 2016, ApJL, 826, L6 Gehrels, N., Chincarini, G., Giommi, P., et al. 2004, ApJ, 611, 1005 Hurley, K., Mitrofanov, I., Kozyrev, A., et al. 2006, ApJS, 164, 124 Loeb, A. 2016, ApJL, 819, L21

Meegan, C., Lichti, G., Bhat, P. N., et al. 2009, ApJ, 702, 791

Rau, A., von Kienlin, A., Hurley, K., \& Lichti, G. 2005, A\&A, 438, 1175

Savchenko, V., Ferrigno, C., Mereghetti, S., et al. 2016, ApJL, 820, L36

Smith, D. M., Lin, R., Turin, P., et al. 2002, SoPh, 210, 33

Svinkin, D., Frederiks, D., Aptekar, R., et al. 2016, ApJS, 224, 10

Woosley, S. 2016, ApJL, 824, L10 